\title{
Tipología Empírica de Adolescentes Consumidores de Drogas según el MACI
}

\author{
Empirical Typology of Adolescent Drug Users \\ According to the MACI
}

\author{
Ximena Faúndez \\ Pontificia Universidad Católica de Chile
}

\author{
Eugenia V. Vinet \\ Universidad de La Frontera
}

\begin{abstract}
Se presenta una tipología empírica de adolescentes consumidores de drogas desarrollada a partir de las escalas de patrones de personalidad del Inventario Clínico para Adolescentes de Millon (MACI).Se aplicó el MACI y una entrevista clínica semiestructurada a 116 adolescentes chilenos consumidores de drogas, de entre 13 y 19 años, tanto hombres como mujeres. El análisis de conglomerados sugirió una solución óptima de 3 tipos para la muestra de hombres, denominados Transgresor-Poderoso, Subclínico y Oposicionista-Autodegradante. En la muestra de mujeres se encontró una solución de 4 tipos, denominados Oposicionista-Transgresor, Inhibido-Autodegradante, Subclínico y en Riesgo. Esta tipología fue interpretada de acuerdo a los 3 grupos de escalas del MACI y a la información clínica, obteniéndose resultados consistentes con los antecedentes teóricos recabados.
\end{abstract}

Palabras clave: drogas, personalidad, adolescencia, MACI.

\begin{abstract}
An empirical typology of adolescent drug users, using the personality pattern scales from the Millon Adolescent Clinical Inventory (MACI), is presented. The MACI and a clinical interview were applied to 116 Chilean adolescent drug users, between 13 and 19 years of age, both male and female. The cluster analysis results suggested for the male sample a solution composed of 3 personality types, named Unruly-Forceful, Subclinical, and Oppositional-Self Demeaning. The final solution of the female sample was composed of 4 types, named Oppositional-Unruly, Inhibited-Self-Demeaning, Subclinical and at Risk. This typology was interpreted according to the 3 groups of MACI scales and clinical information, obtaining consistent results with the theoretical background.
\end{abstract}

Keywords: drugs, personality, adolescence, MACI.

\section{Introducción}

El alto consumo de drogas y los problemas sociales derivados han hecho que este sea uno de los problemas de salud más importantes con los que se enfrenta la sociedad mundial en nuestra época. Se estima que unos 200 millones de personas en el mundo, un $5 \%$ de la población mundial de 15 a 64 años de edad, han consumido drogas ilícitas por lo menos una vez en el último año (United Nations Office on Drugs and Crime, 2008).

En Chile, el último estudio nacional de drogas en población general informa que las tasas de prevalencia de consumo de alcohol de último mes han aumentado significativamente en el país desde un 39\% en el año 1994, hasta un 57\% en el 2006. El uso de drogas ilícitas en el año 2006 permaneció estable, excepto en el consumo de marihuana, que aumentó en relación al informe del año 2004. Actualmente el consumo de drogas se concentra en la población más joven; las prevalencias más altas y la mayor intensidad en el uso se registran en los jóvenes de 19 a 25 años, siendo la adolescencia la etapa más vulnerable para el inicio del consumo de drogas en Chile (Consejo Nacional para el Control de Estupefacientes [CONACE], 2006). En el último estudio en población escolar se señala que un $15,7 \%$ de los estudiantes a nivel nacional declaran haber consumido

Ximena Faúndez Abarca, Universidad de La Frontera, Temuco, Chile. Estudiante del Doctorado en Psicología de la Pontificia Universidad Católica de Chile.

Eugenia V. Vinet, Universidad de La Frontera, Temuco, Chile.

La correspondencia relativa a este artículo debe ser dirigida a Ximena Faúndez Abarca, Escuela de Psicología, Pontificia Universidad Católica de Chile, Vicuña Mackenna 4860, Macul, Santiago, Chile. E-mail: xlfaunde@uc.cl

Esta investigación contó con financiamiento de la Dirección de Investigación de la Universidad de La Frontera, a través del Proyecto DIUFRO 120612, y presenta resultados de la Tesis de Magíster en Psicología de la primera autora, realizada en dicha universidad. 
marihuana en el último año, mientras que un $35 \%$ reconoce consumo actual de tabaco y un $38 \%$, de alcohol (CONACE, 2008).

Hoy en día, los investigadores plantean una visión multidimensional para comprender comportamientos que, como el uso de drogas, conllevan riesgos para la salud. Esta visión trasciende el enfoque biomédico y adopta una perspectiva psicosocial, incluyendo personalidad, comportamiento y estilo de vida como factores de riesgo (Becoña, 2002).

Sin embargo, existen pocos estudios que aborden la personalidad adolescente como factor de riesgo para el consumo de drogas, probablemente debido a la dificultad que implica su estudio o al supuesto, ampliamente aceptado, que no existe un estilo de personalidad adolescente.

En esta investigación se estudian las características de personalidad de adolescentes consumidores de drogas, planteándose los siguientes objetivos: (a) determinar diferencias entre este grupo y otro grupo de adolescentes sin antecedentes de consumo de drogas ni de otros problemas psicológicos; (b) construir una tipología empírica de adolescentes consumidores de drogas, según las escalas de patrones de personalidad del MACI, considerando la variable género; $\mathrm{y}$ (c) explorar las asociaciones entre los tipos empíricos que se establecen y un conjunto de variables psicológicas y sociodemográficas características de la muestra estudiada.

\section{Antecedentes}

\section{Adolescencia, Personalidad y Uso de Drogas}

Estos tres conceptos se relacionan de una manera compleja. La adolescencia es una etapa crítica del desarrollo, en la que el joven se ve enfrentado a múltiples demandas. En esta etapa se reorganizan las estructuras cognitivas y de personalidad del adolescente que darán paso a nuevos comportamientos que le permitan afrontar las demandas y adaptarse a cada nueva situación. Cuando hay incapacidad para afrontar las demandas, el adolescente puede desarrollar comportamientos desadaptativos, más o menos transitorios, y/o problemas psicológicos más graves, producidos por el fracaso persistente de los mecanismos adaptativos
(Ávila, Jiménez \& González, 1996).

Aunque no existe consenso sobre la aplicabilidad del constructo de personalidad en la adolescencia, Millon (1998) postula que esta puede ser descrita en términos de estilos emergentes de personalidad, precursores de los patrones de personalidad del adulto, los cuales derivan de una combinación de las polaridades placer-dolor, activo-pasivo y sí mismo-otros. El modelo teórico de Millon permite comprender el desarrollo y construcción de la personalidad y facilita su clasificación. De este derivan instrumentos de evaluación psicológica, entre los que se encuentra el MACI.

El MACI evalúa estilos de personalidad, preocupaciones psicológicas y psicopatología en adolescentes. Los estilos de personalidad son concebidos como modos de funcionamiento psicológico relativamente estables que surgen a través del desarrollo infantil y se estabilizan en la adolescencia como precursores del estilo de personalidad adulta. Las preocupaciones psicológicas se relacionan con aspectos significativos del desarrollo del adolescente e incluyen características del desarrollo de la identidad, corporalidad, sexualidad y relaciones sociales con los pares, familia y sociedad mayor. El área psicopatológica incluye los trastornos afectivos y de la adaptación social que tienen mayor prevalencia en la adolescencia.

La población adolescente, aunque no registra las tasas más altas de consumo, es considerada la más vulnerable a consumir drogas. Las motivaciones ligadas al consumo en la adolescencia son, en primer lugar, la curiosidad y, luego, el placer. Los que continúan el consumo indican una tercera gran motivación, la del "soporte artificial", fenómeno que se presenta en jóvenes que no encuentran en su vida personal o relacional los medios para relajarse, y solo lo hacen mediante un producto químico que les aporta los espacios y los recursos necesarios, ausentes en sus vidas de forma natural (CONACE, 2004a).

Respecto del género, algunas investigaciones informan que los varones son más precoces y presentan mayor consumo habitual que las mujeres (Kumpfer \& Turner, 1990; Novacek, Raskin \& Hogan, 1991); sin embargo, estas diferencias han ido disminuyendo a través del tiempo y para algunas 
sustancias el género ya no es una variable discriminante en el consumo.

En Chile, la droga ilícita de mayor consumo es la marihuana, con un aumento de la participación femenina en su consumo durante la última década. La razón de 1:4 en el año 1994 (por cada 4 hombres había una mujer que consumía marihuana) ha ido disminuyendo hasta llegar a 1:2 en el año 2006 (CONACE, 2006).

\section{Tipologías de Personalidad de Adolescentes Consumidores de Drogas}

La variabilidad encontrada en las características de personalidad de los consumidores ha llevado a los investigadores a realizar aproximaciones basadas en métodos estadísticos de agrupamiento que permiten el desarrollo de tipologías empíricas. El análisis de conglomerados es un grupo de técnicas multivariantes cuyo principal propósito es agrupar objetos (es decir, personas, productos u otras entidades) en tipos o grupos, basándose en las características que poseen. Los tipos resultantes deben mostrar un alto grado de homogeneidad interna y un alto grado de heterogeneidad externa (Hair \& Anderson, 1999).

La primera tipología desarrollada a partir del MACI surge en Estados Unidos con el trabajo de Romm, Bockian y Harvey (1999) en población clínica. En el área de la delincuencia juvenil, destacan los trabajos de Stefurak, Calhoun y Glaser (2004) y Taylor, Kemper, Loney y Kistner (2006). En Chile se han desarrollado tipologías de personalidad mediante el MACI en población clínica (Díaz, 2003), infractores de ley (Alarcón, Vinet \& Salvo, 2005) y adolescentes en riesgo (Vinet, Salvo \& Forns, 2005).

En el área del consumo de drogas, existen pocos estudios de tipologías de personalidad en adolescentes desarrollados con el MACI. Estos tienen como antecedente el estudio de Grilo, Fehon, Walker y Martino (1996) en Estados Unidos que, aunque no desarrollaron una tipología, compararon los perfiles MACI de una muestra de 44 adolescentes hospitalizados con trastornos por consumo de sustancia (TCS) con los de una muestra de 61 adolescentes sin trastornos por consumo de sustancia (STCS), encontrando diferencias significativas $(p<0,05)$ en 14 de las 31 es- calas. Los adolescentes con TCS mostraron niveles más altos de predisposición delictual y niveles inferiores de ansiedad. Además, se encontró que los adolescentes con TCS se caracterizaron por presentar niveles más altos de indocilidad e insensibilidad social y niveles inferiores de sumisión.

En Chile, Alarcón, Faúndez, Hita y Rodríguez (2004) desarrollaron una tipología de adolescentes consumidores a partir de las escalas de patrones de personalidad del MACI, al evaluar a 41 adolescentes, hombres y mujeres, de 13 a 19 años. Encontraron que la solución de cuatro tipos era la que mejor representaba a los datos. El Tipo 1, denominado Inhibido Emocionalmente, presentó elevaciones en las escalas Autodegradante, Afligido, Introvertido, Inhibido y Sumiso. El Tipo 2, Oposicionista Transgresor, presentó elevaciones en las escalas Transgresor, Poderoso, Oposicionista y Tendencia Borderline. El Tipo 3, denominado Subclínico por presentar gran similitud con la muestra pareada normal, presentó elevaciones en las escalas Conformista y Sumiso. El Tipo 4, denominado Egoísta Dramatizador, presentó elevaciones en las escalas de igual nombre.

El presente estudio continúa la investigación anterior pero, a diferencia de esta, realiza la descripción tipológica considerando el género de los participantes; además, las tipologías son descritas considerando los otros dos grupos de escalas del MACI, la sintomatología clínica y el diagnóstico de consumo de drogas.

\section{Método}

Se utilizó una metodología cuantitativa, con un diseño descriptivo correlacional de grupos independientes.

\section{Participantes}

La población la constituyen los adolescentes, entre 13 y 19 años de edad, consultantes por problemas psicológicos que son consumidores de drogas, procedentes de centros de salud, de hogares de protección y de liceos de alto riesgo, de las regiones Metropolitana, La Araucanía, Los Ríos y Los Lagos.

La muestra de estudio estuvo formada por aquellos adolescentes que en una entrevista clínica informaron consumo de alcohol 
y/o drogas. Ellos fueron 116 adolescentes, 71 hombres con un promedio de 16,1 años de edad y 9,4 años de escolaridad y $45 \mathrm{mu}$ jeres con un promedio de 16,3 años de edad y 9,8 años de escolaridad.

Adicionalmente, se trabajó con una muestra de contraste, obtenida por muestreo no probabilístico por conveniencia, compuesta por 407 adolescentes no consultantes y adaptados al sistema escolar, 201 hombres, con un promedio de 15,9 años de edad y 10,5 años de escolaridad, y 206 mujeres, con un promedio de 15,8 años de edad y 10,6 años de educación, procedentes de las regiones del Bío Bío y La Araucanía.

Las variables edad y escolaridad de las muestras de estudio y contraste fueron comparadas mediante la prueba $t$ de Student para muestras independientes según género, encontrándose que tanto hombres como mujeres presentan diferencias significativas en la variable educación $(p<0,05)$ pero no en la variable edad.

\section{Instrumentos}

El MACI. Es un auto informe desarrollado en Estados Unidos que posee una versión chilena (Vinet et al., 1999). Está dirigido a jóvenes entre 13 y 19 años y consta de 160 afirmaciones a responder en formato verdaderofalso, dependiendo de si ellas representan o no aspectos vinculados a la experiencia personal. Las afirmaciones aportan a 31 escalas:

Doce escalas de Patrones de Personalidad que revelan los estilos de personalidad que surgen durante el desarrollo infantil y se estabilizan en la adolescencia. Ellas son: Introvertido (1), Inhibido (2), Afligido (2B), Sumiso (3), Dramatizador (4), Egoísta (5), Transgresor (6A), Poderoso (6B), Conformista (7), Oposicionista (8A), Autodegradante (8B) y Tendencia Borderline (9).

Ocho escalas de Preocupaciones Expresadas que se focalizan en áreas del desarrollo adolescente: Difusión de la Identidad (A), Autodevaluación (B), Desaprobación Corporal (C), Incomodidad Sexual (D), Inseguridad Grupal (E), Insensibilidad Social (F), Discordia Familiar (G) y Abuso Infantil $(\mathrm{H})$.

Siete escalas de Síndromes Clínicos, que involucran trastornos de alta prevalencia en los adolescentes: Disfunción de la
Alimentación (AA), Tendencia al Abuso de Sustancias (BB), Predisposición Delictual (CC), Tendencia a la Impulsividad (DD), Sentimientos Ansiosos (EE), Afecto Depresivo (FF) y Tendencia Suicida (GG).

Tres escalas Modificadoras que permiten controlar ciertas tendencias de respuesta: Sinceridad (X), Deseabilidad (Y) y Depreciación (Z); y una escala de Validez del protocolo.

En Chile el MACI ha presentado características psicométricas adecuadas. Vinet y Alarcón (2003) reportan que con muestras de adolescentes chilenos, normales y consultantes en salud mental, la consistencia interna de las escalas del MACI fue adecuada y comparable con la reportada en la literatura internacional: en el grupo normal, valores de alfa de Cronbach entre 0,54 y 0,90 , con una mediana de 0,79 , y en la muestra clínica, valores entre 0,51 y 0,88 , con una mediana de 0,80 . Por su parte, Alarcón et al. (2004), con una muestra de 41 adolescentes consumidores de drogas, señalan que el MACI es un instrumento adecuado para evaluar características de personalidad en adolescentes consumidores de drogas. La consistencia interna de las escalas en estos adolescentes fue satisfactoria; un 97\% de ellas presentó un alfa de Cronbach mayor que 0,60 , con una mediana de 0,75 . Además, discrimina adecuadamente entre adolescentes consumidores y no consumidores, pues se evidencian diferencias significativas entre estos en 29 de las 30 escalas del MACI.

Entrevista semiestructurada. Esta fue guiada por dos pautas de construcción $a d-h o c$ denominadas Protocolos A y B. El Protocolo A fue creado a partir de la revisión de fichas clínicas de adolescentes en atención psicológica. Se desarrolla a través de dos preguntas abiertas que indagan motivos de consulta e información sobre estructura y dinámica familiar; además, chequea seis áreas de sintomatología, incluyendo síntomas escolares, conductuales, emocionales, físicos, del área sexual y de las relaciones interpersonales (Álvarez \& Restovic, 2003). En el área de síntomas conductuales chequea el consumo de alcohol y drogas. Quienes responden positivamente a estos síntomas deben responder el Protocolo B. Este se basa en los criterios utilizados por el CONACE para el diagnóstico de consumo no problemático 
(experimental, ocasional y habitual) y en los criterios del Manual Diagnóstico y Estadístico de los Trastornos Mentales ([DSM-IV-TR], American Psychiatric Association, 2002) para el diagnóstico de consumo problemático (abuso y dependencia). Específicamente, consta de 25 criterios organizados en categorías de consumo que son indagados a través de la transformación de los criterios a preguntas. La información obtenida permite clasificar a los participantes según las cinco categorías de consumo ya nombradas. Además, permite indagar sobre la edad de inicio, frecuencia y prevalencia del consumo de drogas ${ }^{1}$.

\section{Procedimiento}

El proyecto de investigación DIUFRO 120612 , que permitió la obtención de las muestras de estudio y contrastación, fue revisado y aprobado por el Comité de Ética de la Universidad de La Frontera. Adicionalmente, se solicitó autorización del Servicio de Salud Araucanía Sur y su Comité de Ética Científico para la evaluación de adolescentes consultantes.

Se contactó a profesionales de centros de salud y otros centros de atención de las regiones Metropolitana, La Araucanía, Los Ríos y Los Lagos para obtener la muestra de estudio. En una segunda etapa, se estableció contacto con liceos de alto riesgo de la ciudad de Temuco, de donde los profesores, inspectores y orientadores de los establecimientos derivaron a alumnos con problemas psicológicos. La entrevista permitió corroborar la condición clínica de los participantes e identificar y evaluar el consumo de drogas.

Todos los participantes de este estudio fueron informados sobre los objetivos y procedimientos de la evaluación, asegurándoseles la confidencialidad de la información. La participación fue formalizada a través de la firma de un documento de consentimiento informado.

\section{Análisis de Datos}

Se examinaron todos los protocolos MACI según los criterios de validez propuestos por Millon (1993), luego sus respuestas se codifi-

\footnotetext{
1 La pauta de entrevista, con ambos protocolos, está disponible para su uso, solicitándola a las autoras de este estudio.
}

caron según procedimientos estándares para derivar las puntuaciones directas por escala. Con los Protocolos A y B se utilizaron criterios de construcción ad-hoc que permitieron el análisis estadístico de la información, mediante el software SPSS v.11.5.

Para el análisis de conglomerados, se seleccionaron como variables de agrupación las 12 escalas de Patrones de Personalidad del MACI, pues estas entregan información sobre rasgos caracterológicos relativamente estables que se encuentran a la base de las posibles dificultades personales e interpersonales de los adolescentes (McCann, 1999). Como procedimiento de agrupación se eligió el método de la varianza mínima de Ward, utilizando la distancia euclidiana al cuadrado como medida de similitud.

\section{Resultados}

\section{Caracterización Sociodemográfica}

Los 116 adolescentes consumidores de drogas (71 hombres y 45 mujeres) proceden de programas de salud mental públicos $(n=58)$, liceos de alto riesgo $(n=31)$, centros de evaluación y rehabilitación para jóvenes infractores de ley $(n=18)$ y hogares para adolescentes en riesgo psicosocial $(n=9)$. Su nivel educacional se distribuyó entre $4^{\circ}$ año básico y $2^{\circ}$ año de educación superior, encontrándose un $85 \%$ en educación media. Del total de la muestra, 21 habían desertado del sistema educacional.

\section{Prevalencia de Consumo}

Se encontró que un $97,4 \%$ de la muestra consumía tabaco y un $99,1 \%$, alcohol. Entre las drogas ilícitas, la marihuana presentó el mayor consumo, con un $74,1 \%$ de la muestra total, seguida por el consumo de solventes volátiles, con un $27,6 \%$, y de pasta base y/o cocaína, con un $20 \%$.

\section{Diagnóstico de Consumo}

El diagnóstico de consumo se estableció considerando el tipo de consumo más alto por persona. En términos generales, un 39,7\% de la muestra total presentó consumo problemático de drogas, correspondiendo a un 42,3\% de los hombres y a un $35,6 \%$ de las mujeres. 
Perfiles de Personalidad de la Muestra de Estudio

En relación al primer objetivo, se determinaron las diferencias de medias entre las puntuaciones MACI obtenidas por los adolescentes con consumo y la muestra de no consumidores. En los hombres se evidencia- ron diferencias significativas en 28 de las 30 escalas, y en las mujeres se encontraron diferencias significativas en las 30 escalas del MACI (ver Tablas 1 y 2).

Se encontró que los adolescentes consumidores presentan puntajes más altos que los no consumidores en la mayoría de las escalas MACI, excepto en las escalas Sumi-

Tabla 1

Estadísticos Descriptivos y Comparación entre Hombres Consumidores y No Consumidores en las Escalas MACI

\begin{tabular}{|c|c|c|c|c|c|c|}
\hline \multirow{2}{*}{ Escalas MACI } & \multicolumn{2}{|c|}{ Consumidores } & \multicolumn{2}{|c|}{ No consumidores } & \multirow{2}{*}{$t$} & \multirow{2}{*}{$p$} \\
\hline & $M$ & $D S$ & $M$ & $D S$ & & \\
\hline \multicolumn{7}{|c|}{ Patrones de Personalidad } \\
\hline 1 & 25,10 & 10,10 & 21,10 & 8,12 & 3,01 & $* * *$ \\
\hline $2 \mathrm{~A}$ & 20,76 & 9,67 & 17,02 & 7,88 & 2,93 & $* * *$ \\
\hline $2 \mathrm{~B}$ & 17,97 & 9,97 & 8,36 & 6,30 & 7,61 & $* * *$ \\
\hline 3 & 39,35 & 10,20 & 48,81 & 7,63 & $-7,14$ & $* * *$ \\
\hline 4 & 42,07 & 8,72 & 41,61 & 7,80 & 0,41 & \\
\hline 5 & 36,86 & 8,99 & 38,72 & 7,79 & $-1,66$ & * \\
\hline $6 \mathrm{~A}$ & 43,48 & 10,09 & 26,93 & 8,45 & 2,38 & $* * *$ \\
\hline $6 \mathrm{~B}$ & 18,68 & 7,99 & 9,30 & 5,54 & 9,14 & $* * *$ \\
\hline 7 & 37,90 & 9,40 & 50,81 & 6,61 & $-10,66$ & $* * *$ \\
\hline $8 \mathrm{~A}$ & 29,24 & 10,12 & 16,52 & 7,22 & 9,75 & $* * *$ \\
\hline $8 \mathrm{~B}$ & 26,20 & 13,72 & 12,75 & 9,03 & 7,69 & $* * *$ \\
\hline 9 & 16,99 & 8,09 & 9,01 & 4,99 & 7,80 & $* * *$ \\
\hline \multicolumn{7}{|c|}{ Preocupaciones Expresadas } \\
\hline $\mathrm{A}$ & 19,01 & 7,88 & 11,44 & 5,53 & 7,47 & $* * *$ \\
\hline $\mathrm{B}$ & 24,92 & 13,39 & 13,51 & 9,44 & 6,62 & $* * *$ \\
\hline $\mathrm{C}$ & 8,07 & 7,43 & 5,05 & 4,99 & 3,17 & $* * *$ \\
\hline $\mathrm{D}$ & 24,44 & 5,89 & 30,72 & 5,54 & $-8,08$ & $* * *$ \\
\hline $\mathrm{E}$ & 8,96 & 5,65 & 8,44 & 4,02 & 0,71 & \\
\hline $\mathrm{F}$ & 33,49 & 8,82 & 27,86 & 6,87 & 4,89 & $* * *$ \\
\hline $\mathrm{G}$ & 21,55 & 7,34 & 12,95 & 4,28 & 9,32 & $* * *$ \\
\hline $\mathrm{H}$ & 8,96 & 5,89 & 4,00 & 3,68 & 6,65 & $* * *$ \\
\hline \multicolumn{7}{|c|}{ Síndromes Clínicos } \\
\hline $\mathrm{AA}$ & 9,39 & 9,18 & 5,53 & 5,24 & 3,36 & $* * *$ \\
\hline $\mathrm{BB}$ & 29,89 & 11,04 & 10,47 & 6,87 & 13,90 & $* * *$ \\
\hline $\mathrm{CC}$ & 31,75 & 8,21 & 23,21 & 5,21 & 8,19 & $* * *$ \\
\hline DD & 22,21 & 6,11 & 14,92 & 5,36 & 9,49 & $* * *$ \\
\hline $\mathrm{EE}$ & 23,30 & 6,62 & 31,09 & 5,61 & $-9,60$ & $* * *$ \\
\hline $\mathrm{FF}$ & 16,97 & 11,17 & 10,86 & 6,49 & 4,36 & $* * *$ \\
\hline GG & 11,83 & 9,17 & 3,63 & 4,01 & 7,30 & $* * *$ \\
\hline \multicolumn{7}{|c|}{ Escalas Modificadoras } \\
\hline $\mathrm{X}$ & 380,06 & 70,34 & 295,57 & 48,23 & 9,37 & $* * *$ \\
\hline $\mathrm{Y}$ & 11,31 & 2,95 & 12,53 & 2,37 & $-3,15$ & $* * *$ \\
\hline $\mathrm{Z}$ & 5,56 & 3,76 & 2,41 & 2,25 & 6,67 & $* * *$ \\
\hline
\end{tabular}

${ }^{*} p<0,05 ; * * p<0,01 ; * * * p<0,001$. 
so (3), Egoísta (5), Conformista (7), Incomodidad Sexual (D) y Sentimientos Ansiosos (EE). Interpretativamente, esto significa que los adolescentes consumidores presentan un estilo más rebelde y desafiante de los valores y preceptos culturales que los no consumidores; son menos auto-centrados y manifiestan menor ansiedad y menores dificultades que el resto de los jóvenes chilenos en el enfrentamiento de su propia sexualidad.

\section{Tipologías de Consumidores}

La elección del número de conglomerados se basó en la observación y análisis del dendograma, los gráficos de perfiles y los an-

Tabla 2

Estadisticos Descriptivos y Comparación entre Mujeres Consumidoras y No Consumidoras en las Escalas MACI

\begin{tabular}{|c|c|c|c|c|c|c|}
\hline \multirow{2}{*}{ Escalas MACI } & \multicolumn{2}{|c|}{ Consumidoras } & \multicolumn{2}{|c|}{ No consumidoras } & \multirow{2}{*}{$t$} & \multirow{2}{*}{$p$} \\
\hline & $M$ & $D S$ & $M$ & $D S$ & & \\
\hline \multicolumn{7}{|c|}{ Patrones de Personalidad } \\
\hline 1 & 33,20 & 11,01 & 20,27 & 7,66 & 7,49 & $* * *$ \\
\hline $2 \mathrm{~A}$ & 32,82 & 12,02 & 19,98 & 7,85 & 6,85 & $* * *$ \\
\hline $2 \mathrm{~B}$ & 27,80 & 12,40 & 10,25 & 7,95 & 9,10 & $* * *$ \\
\hline 3 & 45,38 & 12,14 & 54,93 & 7,72 & $-5,06$ & $* * *$ \\
\hline 4 & 30,07 & 10,44 & 39,68 & 7,74 & $-5,84$ & $* * *$ \\
\hline 5 & 25,31 & 10,01 & 34,73 & 8,91 & $-6,29$ & $* * *$ \\
\hline $6 \mathrm{~A}$ & 37,69 & 11,68 & 22,14 & 8,73 & 8,43 & $* * *$ \\
\hline $6 \mathrm{~B}$ & 17,71 & 9,36 & 7,08 & 4,69 & 7,42 & $* * *$ \\
\hline 7 & 33,98 & 12,16 & 52,26 & 6,90 & $-9,75$ & $* * *$ \\
\hline $8 \mathrm{~A}$ & 35,91 & 13,78 & 15,09 & 8,27 & 9,76 & $* * *$ \\
\hline $8 \mathrm{~B}$ & 39,98 & 15,77 & 16,27 & 10,95 & 9,59 & $* * *$ \\
\hline 9 & 24,18 & 9,39 & 10,28 & 6,91 & 9,39 & $* * *$ \\
\hline \multicolumn{7}{|c|}{ Preocupaciones Expresadas } \\
\hline $\mathrm{A}$ & 23,96 & 9,18 & 11,82 & 6,62 & 8,40 & $* * *$ \\
\hline $\mathrm{B}$ & 38,98 & 15,94 & 18,19 & 11,34 & 8,30 & $* * *$ \\
\hline $\mathrm{C}$ & 18,69 & 8,53 & 10,53 & 7,68 & 6,33 & $* * *$ \\
\hline $\mathrm{D}$ & 31,29 & 5,42 & 35,19 & 4,98 & $-4,69$ & $* * *$ \\
\hline $\mathrm{E}$ & 12,98 & 7,84 & 8,35 & 3,19 & 3,89 & $* * *$ \\
\hline $\mathrm{F}$ & 25,18 & 9,97 & 21,70 & 6,74 & 2,23 & $*$ \\
\hline $\mathrm{G}$ & 24,20 & 8,32 & 13,33 & 5,35 & 8,40 & $* * *$ \\
\hline $\mathrm{H}$ & 17,44 & 8,86 & 5,52 & 4,64 & 8,77 & $* * *$ \\
\hline \multicolumn{7}{|c|}{ Síndromes Clínicos } \\
\hline $\mathrm{AA}$ & 22,31 & 10,51 & 13,31 & 9,32 & 5,74 & $* * *$ \\
\hline $\mathrm{BB}$ & 27,31 & 13,72 & 7,54 & 5,78 & 9,49 & $* * *$ \\
\hline $\mathrm{CC}$ & 23,16 & 7,53 & 18,48 & 4,24 & 4,03 & $* * *$ \\
\hline DD & 21,56 & 7,05 & 12,74 & 5,80 & 7,83 & $* * *$ \\
\hline $\mathrm{EE}$ & 29,64 & 8,35 & 35,92 & 5,33 & $-4,84$ & $* * *$ \\
\hline $\mathrm{FF}$ & 29,49 & 12,28 & 15,38 & 7,83 & 7,38 & $* * *$ \\
\hline GG & 21,69 & 10,99 & 5,67 & 5,99 & 9,47 & $* * *$ \\
\hline \multicolumn{7}{|c|}{ Escalas Modificadoras } \\
\hline $\mathrm{X}$ & 423,25 & 86,62 & 293,16 & 50,46 & 9,72 & $* * *$ \\
\hline $\mathrm{Y}$ & 9,29 & 3,35 & 11,86 & 2,51 & $-4,87$ & $* * *$ \\
\hline $\mathrm{Z}$ & 9,16 & 4,50 & 3,46 & 2,70 & 8,17 & $* * *$ \\
\hline
\end{tabular}

$* p<0,05 ; * * p<0,01 ; * * * p<0,001$. 
tecedentes teóricos aportados por Alarcón et al. (2004) y Grilo et al. (1996). En los hombres se decidió que la agrupación que mejor representaba los datos de Patrones de Personalidad era la solución de tres tipos; en las mujeres se optó por una solución de cuatro tipos. Ambas soluciones maximizan la homogeneidad interna y la heterogeneidad externa entre los grupos. En las Tablas 3 y 4 se presentan las medias obtenidas en cada uno de los tipos de hombres y mujeres, respectivamente, y las diferencias entre ellas.

\section{Interpretación de la Tipología de Hombres}

Cada tipo fue interpretado psicológicamente, teniendo como referencia el perfil obtenido por los no consumidores de la muestra

Tabla 3

Comparación de Medias de las Escalas MACI según Tipos en los Hombres Consumidores

\begin{tabular}{|c|c|c|c|c|c|c|c|c|}
\hline \multirow{3}{*}{ Escalas MACI } & \multirow{2}{*}{\multicolumn{2}{|c|}{$\begin{array}{c}\text { Tipo } 1 \\
(n=20)\end{array}$}} & \multirow{2}{*}{\multicolumn{2}{|c|}{$\begin{array}{c}\text { Tipo } 2 \\
(n=21)\end{array}$}} & \multirow{2}{*}{\multicolumn{2}{|c|}{$\begin{array}{c}\text { Tipo } 3 \\
(n=30) \\
\end{array}$}} & \multirow{3}{*}{$F$} & \multirow{3}{*}{$p$} \\
\hline & & & & & & & & \\
\hline & $M$ & $D S$ & $M$ & $D S$ & $M$ & $D S$ & & \\
\hline \multicolumn{9}{|c|}{ Patrones de Personalidad } \\
\hline 1 & $20,60_{\mathrm{a}}$ & 6,91 & $18,19_{\mathrm{a}}$ & 6,87 & $32,93_{\mathrm{b}}$ & 8,39 & 28,38 & $* * *$ \\
\hline $2 \mathrm{~A}$ & $13,95_{\mathrm{a}}$ & 5,76 & $16,00_{\mathrm{a}}$ & 6,28 & $28,63_{\mathrm{b}}$ & 8,02 & 33,78 & $* * *$ \\
\hline $2 \mathrm{~B}$ & $12,90_{\mathrm{a}}$ & 5,16 & $9,62_{\mathrm{a}}$ & 6,02 & $27,20_{\mathrm{b}}$ & 6,42 & 63,56 & $* * *$ \\
\hline 3 & $31,90_{\mathrm{a}}$ & 7,36 & $44,86_{\mathrm{b}}$ & 10,62 & $40,47_{\mathrm{b}}$ & 8,73 & 11,04 & $* * *$ \\
\hline 4 & $47,75_{\mathrm{a}}$ & 4,38 & $43,95_{\mathrm{a}}$ & 6,04 & $36,97_{\mathrm{b}}$ & 9,70 & 13,36 & $* * *$ \\
\hline 5 & $41,70_{\mathrm{a}}$ & 6,17 & $40,19_{\mathrm{a}}$ & 8,22 & $31,30_{b}$ & 8,22 & 13,74 & $* * *$ \\
\hline $6 \mathrm{~A}$ & $51,95_{\mathrm{a}}$ & 6,23 & $34,00_{\mathrm{b}}$ & 8,11 & $44,47_{\mathrm{c}}$ & 7,70 & 30,22 & $* * *$ \\
\hline $6 \mathrm{~B}$ & $23,00_{\mathrm{a}}$ & 5,75 & $11,52_{\mathrm{b}}$ & 4,91 & $20,80_{\mathrm{a}}$ & 7,83 & 18,66 & $* * *$ \\
\hline 7 & $34,55_{\mathrm{a}}$ & 4,54 & $48,67_{\mathrm{b}}$ & 6,41 & $32,60_{\mathrm{a}}$ & 7,15 & 44,18 & $* * *$ \\
\hline $8 \mathrm{~A}$ & $30,85_{\mathrm{a}}$ & 4,22 & $17,52_{\mathrm{b}}$ & 4,70 & $36,37_{\mathrm{c}}$ & 8,17 & 55,84 & $* * *$ \\
\hline $8 \mathrm{~B}$ & $19,05_{\mathrm{a}}$ & 6,91 & $14,38_{\mathrm{a}}$ & 8,90 & $39,23_{\mathrm{b}}$ & 7,51 & 74,61 & $* * *$ \\
\hline 9 & $15,25_{\mathrm{a}}$ & 3,82 & $8,57_{\mathrm{b}}$ & 4,59 & $24,03_{\mathrm{c}}$ & 5,40 & 66,80 & $* * *$ \\
\hline \multicolumn{9}{|c|}{ Preocupaciones Expresadas } \\
\hline A & $18,35_{\mathrm{a}}$ & 5,96 & $11,43_{\mathrm{b}}$ & 4,82 & $24,77_{\text {c }}$ & 5,88 & 35,09 & $* * *$ \\
\hline B & $17,20_{\mathrm{a}}$ & 6,70 & $15,19_{\mathrm{a}}$ & 10,58 & $36,87_{b}$ & 8,31 & 49,80 & $* * *$ \\
\hline $\mathrm{C}$ & $4,35_{\mathrm{a}}$ & 3,31 & $4,67_{\mathrm{a}}$ & 4,66 & $12,93_{\mathrm{b}}$ & 8,30 & 15,84 & $* * *$ \\
\hline $\mathrm{D}$ & $23,10_{\mathrm{a}}$ & 4,73 & $27,10_{\mathrm{a}}$ & 4,23 & $23,47_{\mathrm{a}}$ & 7,03 & 3,26 & \\
\hline $\mathrm{E}$ & $6,50_{\mathrm{a}}$ & 3,98 & $8,29_{\mathrm{ab}}$ & 3,76 & $11,07_{\mathrm{b}}$ & 6,91 & 4,55 & $* *$ \\
\hline $\mathrm{F}$ & $41,20_{\mathrm{a}}$ & 4,81 & $30,67_{\mathrm{b}}$ & 7,75 & $30,33_{\mathrm{b}}$ & 8,61 & 14,86 & $* * *$ \\
\hline $\mathrm{G}$ & $22,45_{\mathrm{a}}$ & 4,45 & $14,90_{\mathrm{b}}$ & 4,91 & $25,60_{\mathrm{a}}$ & 7,15 & 20,86 & $* * *$ \\
\hline $\mathrm{H}$ & $6,20_{\mathrm{a}}$ & 2,90 & $5,10_{\mathrm{a}}$ & 3,13 & $13,50_{b}$ & 5,83 & 27,50 & $* * *$ \\
\hline \multicolumn{9}{|c|}{ Síndromes Clínicos } \\
\hline $\mathrm{AA}$ & $5,00_{\mathrm{a}}$ & 4,27 & $4,90_{\mathrm{a}}$ & 4,09 & $15,47_{\mathrm{b}}$ & 10,68 & 16,34 & $* * *$ \\
\hline $\mathrm{BB}$ & $35,95_{\mathrm{a}}$ & 6,96 & $18,86_{b}$ & 9,13 & $33,57_{\mathrm{a}}$ & 8,82 & 25,96 & $* * *$ \\
\hline $\mathrm{CC}$ & $39,80_{\mathrm{a}}$ & 4,34 & $28,81_{b}$ & 7,45 & $28,43_{b}$ & 7,07 & 21,06 & $* * *$ \\
\hline DD & $26,10_{\text {a }}$ & 4,63 & $16,19_{\mathrm{b}}$ & 3,57 & $23,83_{\mathrm{a}}$ & 5,29 & 26,37 & $* * *$ \\
\hline $\mathrm{EE}$ & $17,20_{\mathrm{a}}$ & 4,81 & $26,67_{\mathrm{b}}$ & 6,77 & $25,00_{\mathrm{b}}$ & 4,69 & 18,21 & $* * *$ \\
\hline $\mathrm{FF}$ & $8,40_{a}$ & 3,94 & $10,52_{\mathrm{a}}$ & 7,77 & $27,20_{b}$ & 7,76 & 57,40 & $* * *$ \\
\hline GG & $7,55_{\mathrm{a}}$ & 5,10 & $5,05_{\mathrm{a}}$ & 4,27 & $19,43_{b}$ & 8,22 & 37,01 & $* * *$ \\
\hline
\end{tabular}

Nota. Las medias con distinto subíndice son significativamente diferentes ( $p<0,05$, prueba post hoc de Tukey). ${ }^{*} p<0,05 ; * * p<0,01 ; * * * p<0,001$. 
de contrastación. A través de las figuras que acompañan la interpretación, se puede observar el perfil de las escalas de Patrones de Personalidad obtenido por cada tipo con respecto al perfil de contrastación de adolescentes no consumidores y sin patología evidente.

Perfil tipo 1, hombres Transgresores Poderosos. Corresponde al $28,2 \%$ de la mues- tra de hombres. Su perfil se caracteriza, como se aprecia en la Figura 1, por elevaciones significativas en cuatro escalas: 4, $6 \mathrm{~A}, 6 \mathrm{~B}$ y $8 \mathrm{~A}$.

La elevación en Dramatizador (4) indica que estos jóvenes asumen una postura de dependencia activa, alcanzando sus objetivos a través de la manipulación, seducción y gregarismo. La elevación conjunta en

Tabla 4

Comparación de Medias de las Escalas MACI según Tipos en las Mujeres Consumidoras

\begin{tabular}{|c|c|c|c|c|c|c|c|c|c|c|}
\hline \multirow[t]{2}{*}{ Escalas MACI } & \multicolumn{2}{|c|}{$\begin{array}{c}\text { Tipo } 1 \\
(n=13)\end{array}$} & \multicolumn{2}{|c|}{$\begin{array}{c}\text { Tipo } 2 \\
(n=11)\end{array}$} & \multicolumn{2}{|c|}{$\begin{array}{c}\text { Tipo } 3 \\
(n=12)\end{array}$} & \multicolumn{2}{|c|}{$\begin{array}{l}\text { Tipo } 4 \\
(n=9)\end{array}$} & \multirow[t]{2}{*}{$F$} & \multirow[t]{2}{*}{$p$} \\
\hline & $M$ & $D S$ & $M$ & $D S$ & $M$ & $D S$ & $M$ & $D S$ & & \\
\hline \multicolumn{11}{|c|}{ P. de Personalidad } \\
\hline 1 & $37,08_{\mathrm{ab}}$ & 7,39 & $42,45_{\mathrm{b}}$ & 7,79 & $19,92_{\mathrm{c}}$ & 5,98 & $34,00_{\mathrm{a}}$ & 7,26 & 21,46 & $* * *$ \\
\hline $2 \mathrm{~A}$ & $35,54_{\mathrm{ab}}$ & 8,87 & $44,45_{\mathrm{b}}$ & 9,11 & $19,67_{\mathrm{c}}$ & 5,03 & $32,22_{\mathrm{a}}$ & 8,97 & 18,57 & $* * *$ \\
\hline $2 \mathrm{~B}$ & $36,08_{\mathrm{a}}$ & 5,97 & $38,73_{\mathrm{a}}$ & 5,73 & $13,92_{\mathrm{b}}$ & 6,49 & $21,00_{\mathrm{b}}$ & 8,75 & 36,84 & $* * *$ \\
\hline 3 & $33,77_{\mathrm{a}}$ & 6,81 & $52,00_{\mathrm{b}}$ & 8,43 & $51,25_{\mathrm{b}}$ & 10,12 & $46,22_{\mathrm{b}}$ & 13,41 & 9,47 & $* * *$ \\
\hline 4 & $30,77_{\mathrm{a}}$ & 5,99 & $21,18_{\mathrm{b}}$ & 7,77 & $41,75_{\mathrm{c}}$ & 6,89 & $24,33_{\mathrm{ab}}$ & 7,35 & 19,31 & $* * *$ \\
\hline 5 & $27,15_{\mathrm{a}}^{a}$ & 5,76 & $16,00_{\mathrm{b}}$ & 4,54 & $35,67_{\mathrm{c}}$ & 8,35 & $20,22_{\mathrm{ab}}$ & 8,06 & 18,21 & $* * *$ \\
\hline $6 \mathrm{~A}$ & $52,00_{a}$ & 2,61 & $34,27_{\mathrm{b}}$ & 6,83 & $31,25_{\mathrm{b}}$ & 9,42 & $29,78_{\mathrm{b}}$ & 8,84 & 24,48 & $* * *$ \\
\hline $6 \mathrm{~B}$ & $27,00_{a}$ & 7,54 & $17,09_{\mathrm{b}}$ & 6,40 & $13,00_{b}$ & 7,66 & $11,33_{\mathrm{b}}$ & 6,71 & 11,46 & $* * *$ \\
\hline 7 & $21,85_{a}$ & 5,29 & $29,73_{\mathrm{b}}$ & 7,00 & $47,92_{\mathrm{c}}$ & 6,11 & $38,11_{d}$ & 9,14 & 32,97 & $* * *$ \\
\hline $8 \mathrm{~A}$ & $50,31_{\mathrm{a}}$ & 6,02 & $41,55_{\mathrm{b}}$ & 9,34 & $21,83_{\mathrm{c}}$ & 6,01 & $27,00_{\mathrm{c}}$ & 7,97 & 37,89 & $* * *$ \\
\hline $8 \mathrm{~B}$ & $49,00_{\mathrm{a}}$ & 9,12 & $54,45_{\mathrm{a}}$ & 8,25 & $24,42_{\mathrm{b}}$ & 11,32 & $30,00_{b}$ & 9,27 & 25,94 & $* * *$ \\
\hline 9 & $31,62_{\mathrm{a}}$ & 3,20 & $32,09_{\mathrm{a}}$ & 3,89 & $14,50_{\mathrm{b}}$ & 5,76 & $16,67_{\mathrm{b}}$ & 5,00 & 49,51 & $* * *$ \\
\hline \multicolumn{11}{|l|}{ P. Expresadas } \\
\hline $\mathrm{A}$ & $31,46_{\mathrm{a}}$ & 5,09 & $28,18_{\mathrm{ab}}$ & 4,09 & $12,67_{\mathrm{c}}$ & 5,52 & $23,00_{b}$ & 7,33 & 27,11 & $* * *$ \\
\hline B & $46,77_{\mathrm{a}}$ & 9,41 & $55,00_{\mathrm{a}}$ & 4,86 & $20,83_{\mathrm{b}}$ & 8,98 & $32,33_{\mathrm{c}}$ & 11,39 & 33,75 & $* * *$ \\
\hline $\mathrm{C}$ & $20,46_{\mathrm{ab}}$ & 8,68 & $26,09_{\mathrm{b}}$ & 4,21 & $12,08_{\mathrm{c}}$ & 6,20 & $15,89_{\text {ac }}$ & 7,56 & 8,63 & $* * *$ \\
\hline $\mathrm{D}$ & $27,15_{\mathrm{a}}$ & 5,74 & $31,09_{\mathrm{ab}}$ & 4,11 & $34,58_{\mathrm{b}}$ & 4,98 & $33,11_{\mathrm{b}}$ & 2,98 & 5,76 & $* *$ \\
\hline $\mathrm{E}$ & $13,31_{\mathrm{ab}}$ & 7,22 & $17,09_{\mathrm{b}}$ & 9,18 & $7,17_{\mathrm{a}}$ & 3,27 & $15,22_{\mathrm{ab}}$ & 7,74 & 4,22 & $*$ \\
\hline $\mathrm{F}$ & $31,77_{\mathrm{a}}$ & 6,52 & $17,91_{\mathrm{b}}$ & 4,23 & $27,42_{\mathrm{ab}}$ & 11,07 & $21,56_{\mathrm{b}}$ & 11,26 & 5,93 & $* *$ \\
\hline G & 32,54 a & 5,03 & $25,09_{\mathrm{b}}$ & 5,45 & $18,67_{\mathrm{b}}$ & 6,85 & $18,44_{\mathrm{b}}$ & 6,60 & 14,72 & $* * *$ \\
\hline $\mathrm{H}$ & $21,85_{\mathrm{ab}}$ & 8,27 & $22,82_{\mathrm{a}}$ & 7,35 & $13,83_{\mathrm{bc}}$ & 7,31 & $9,33_{\mathrm{c}}$ & 4,87 & 8,43 & $* * *$ \\
\hline \multicolumn{11}{|l|}{ S. Clínicos } \\
\hline $\mathrm{AA}$ & $24,46_{\mathrm{ab}}$ & 10,49 & $31,82_{\mathrm{b}}$ & 6,16 & $16,83_{\mathrm{a}}$ & 6,87 & $14,89_{\mathrm{a}}$ & 9,52 & 8,86 & $* * *$ \\
\hline $\mathrm{BB}$ & $42,15_{\mathrm{a}}$ & 9,35 & $28,91_{b}$ & 9,38 & $18,08_{c}$ & 9,55 & $16,22_{\mathrm{c}}$ & 6,38 & 21,07 & $* * *$ \\
\hline $\mathrm{CC}$ & $28,92_{\mathrm{a}}$ & 3,77 & $18,64_{\mathrm{b}}$ & 6,20 & $23,75_{\mathrm{ab}}$ & 7,15 & $19,56_{\mathrm{b}}$ & 8,66 & 6,19 & $* *$ \\
\hline DD & $28,77_{\mathrm{a}}$ & 2,17 & $22,36_{\mathrm{b}}$ & 4,63 & $17,42_{\mathrm{bc}}$ & 5,78 & $15,67_{\mathrm{c}}$ & 6,60 & 16,66 & $* * *$ \\
\hline $\mathrm{EE}$ & $21,77_{\mathrm{a}}$ & 3,81 & $34,82_{\mathrm{b}}$ & 7,00 & $31,58_{b}$ & 5,98 & $32,11_{b}$ & 10,04 & 8,80 & $* * *$ \\
\hline $\mathrm{FF}$ & $36,54_{\mathrm{a}}$ & 8,55 & $39,73_{\mathrm{a}}$ & 6,13 & $16,42_{\mathrm{b}}$ & 7,24 & $24,22_{\mathrm{b}}$ & 9,40 & 22,08 & $* * *$ \\
\hline GG & $30,38_{a}$ & 5,64 & $29,55_{\mathrm{a}}$ & 7,12 & $11,92_{\mathrm{b}}$ & 5,93 & $12,56_{\mathrm{b}}$ & 7,81 & 27,59 & $* * *$ \\
\hline
\end{tabular}

Nota. Las medias con distinto subíndice son significativamente diferentes ( $p<0,05$, prueba post hoc de Tukey).

${ }^{*} p<0,05 ;{ }^{* *} p<0,01 ; * * * 00,001$. 


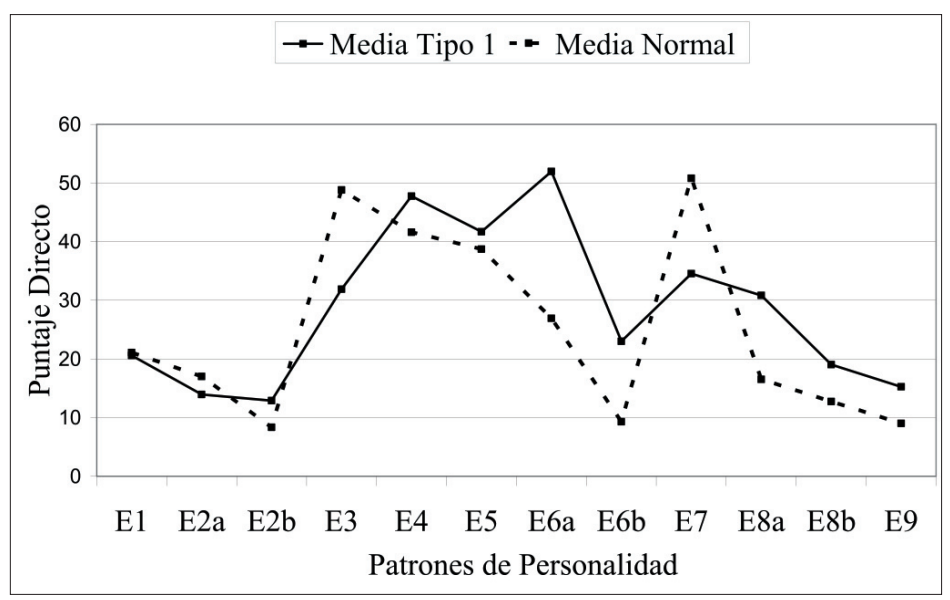

Figura 1. Perfil hombres tipo 1.

Transgresor, Poderoso y Oposicionista (6A6B-8A) indica que presentan conductas hostiles, enojo y falta de relaciones interpersonales estrechas (McCann, 1999), siendo congruente con datos de la entrevista que señalan que un $95 \%$ de este grupo informa mala conducta en el colegio, un $75 \%$ rebeldía y un $70 \%$ agresividad.

En Preocupaciones Expresadas este tipo presenta elevaciones en Difusión de la Identidad (A), Insensibilidad Social (F) y Discordia Familiar (G). En Síndromes Clínicos las elevaciones se presentan en Tendencia al Abuso de Sustancias (BB), Predisposición Delictual (CC) y Tendencia a la Impulsividad (DD). Además, presentan disminución de Sentimientos Ansiosos (EE), coincidiendo con la evaluación clínica que los señala como el grupo que informa menos sintomatología ansiosa. Un 50\% tiene como motivo de consulta principal el consumo de drogas y un $30 \%$, conductas delictivas. Poseen el nivel de consumo de drogas más elevado con un $65 \%$ de consumo problemático (ver Tabla 5).

Perfil tipo 2, Subclínico hombres. Como se aprecia en la Figura 2, este tipo presenta un perfil de personalidad semejante al de los jóvenes no consumidores. En Preocupaciones Expresadas solo presenta una moderada elevación en Incomodidad Sexual (D) y en Síndromes Clínicos aparecen elevaciones en Tendencia al Abuso de Sustancia (BB) y Predisposición Delictual (CC). La Tabla 5 muestra que el $52 \%$ presenta problemas escolares como motivo de consulta principal y un $57 \%$ refiere conductas infantiles e hiperactividad; además, es la agrupación de hombres con menor sintomatología y el me-

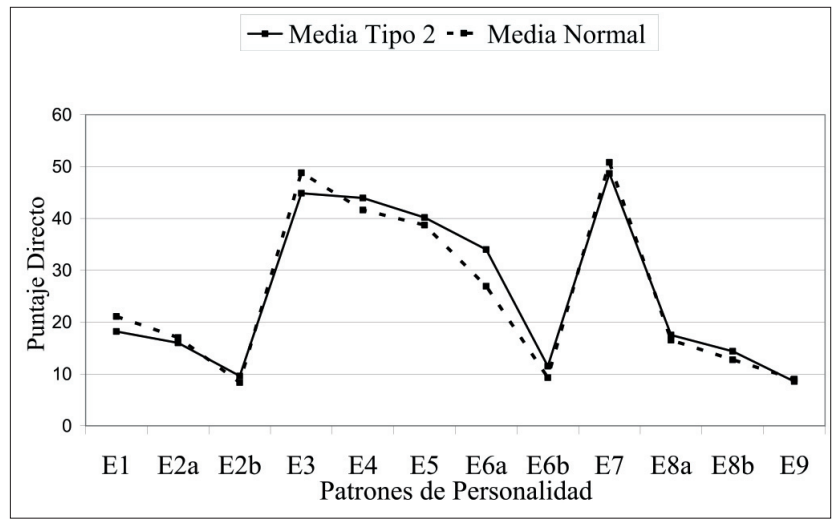

Figura 2. Perfil hombres tipo 2. 
Tabla 5

Motivos de Consulta y Consumo en Hombres y Mujeres, según Tipología

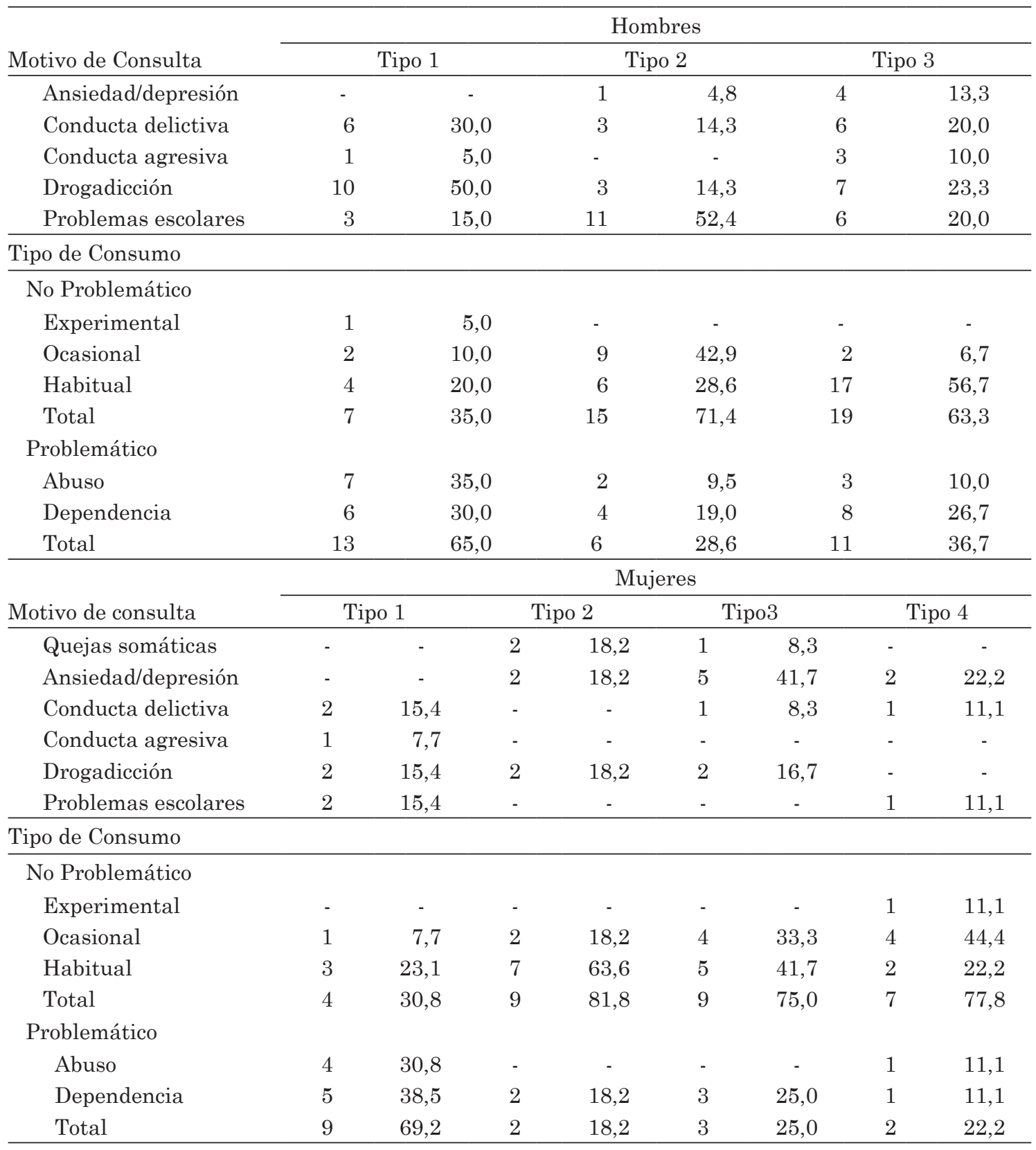

Nota. Los guiones indican que no se encontró dicha información en el tipo. La primera columna de cada tipo expresa la frecuencia absoluta y la segunda, el porcentaje.

nor nivel de consumo de drogas $(71,4 \%$ de consumo no problemático), el cual, al no estar asociado a sintomatología clínica, puede entenderse como una búsqueda de aceptación y fuente de gratificación social.
Perfil tipo 3, hombres Oposicionistas Autodegradantes. Como muestra la Figura 3, este tipo presenta la constelación Introvertido-Inhibido-Afligido (1-2A2B) que identifica a adolescentes con una 


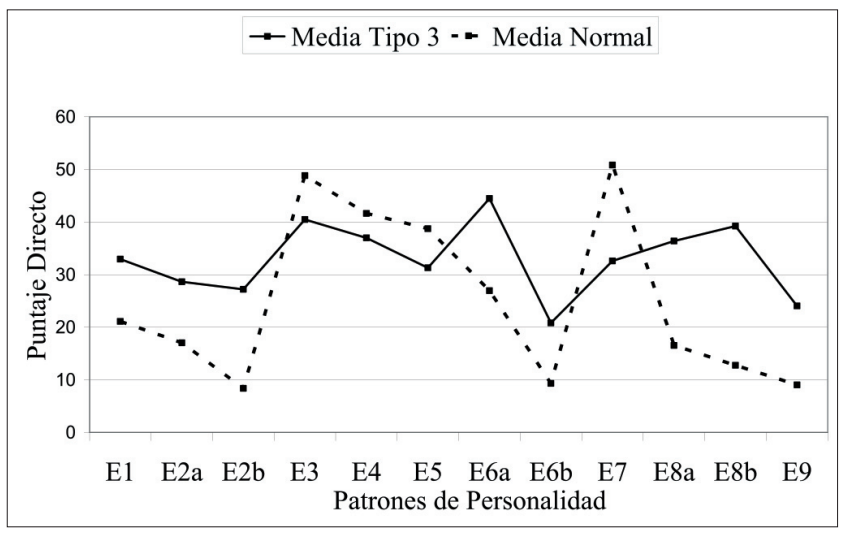

Figura 3. Perfil hombres tipo 3.

marcada dificultad para experimentar vivencias positivas o reforzadoras en su vida cotidiana. Además, la constelación Afligido-Oposicionista-Autodegradante y Tendencia Borderline (2B-8A-8B-9) los caracteriza como adolescentes con disforia y depresión crónica, lo que se manifiesta en un número importante de actitudes y conductas negativas (McCann, 1999).

En Preocupaciones Expresadas este tipo presenta elevaciones de Autodevaluación (B) y Desaprobación Corporal (C), un alto nivel de Inseguridad Grupal (E), percepción de Disfunción Familiar (G) y preocupaciones por experiencias de Abuso Infantil (H). Además, presentan elevaciones en las escalas Disfunciones Alimentarias (AA), Tendencia al Abuso de Sustancias (BB), Predisposición y Delictual (CC), Afecto Depresivo (FF) y Tendencia Suicida (GG). La Tabla 5 muestra que ellos presentan una gran variedad de motivos de consulta y que un $36,7 \%$ presenta consumo problemático, con un alto nivel de dependencia (26,7\%). Según McCann (1999), cuando la elevación en la Tendencia al Abuso de Sustancias (BB) está acompañada de elevaciones en $2 \mathrm{~A}$ y E, el adolescente puede usar sustancias ilícitas para hacer frente a altos niveles de ansiedad social o para ganar aceptación de sus pares.

\section{Interpretación de la Tipología de Mujeres}

Perfil tipo 1, mujeres Oposicionistas Transgresoras. Como se observa en la Figura 4, estas adolescentes presentan la constelación Introvertido-Inhibido-Afligido, que identifica a adolescentes con una marcada dificultad para experimentar vivencias positivas en su vida cotidiana. Comparten con los hombres Transgresores Poderosos la configuración 6A-6B-8A, estilo oposicionista que oscila entre reacciones de obe-

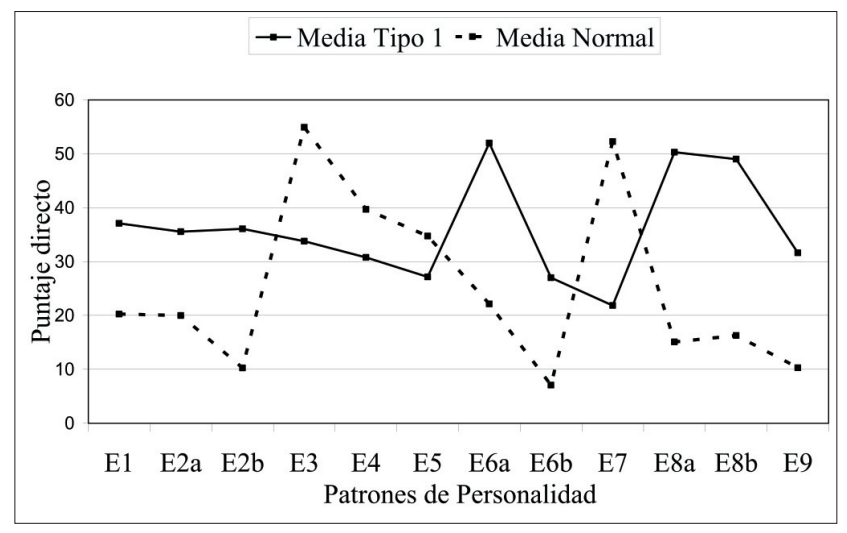

Figura 4. Perfil mujeres tipo 1. 
diencia y desafío. Ellas ejercen poder en las relaciones, son irresponsables y transgresoras de normas, llegando a conductas delictuales; además, presentan alta inestabilidad emocional con arranques de rabia y auto agresiones: el 84,6\% refiere intentos de suicidio.

El conjunto de escalas de Preocupaciones Expresadas evidencia que estas jóvenes presentan importante sintomatología y desajuste psicopatológico. Presentan las mayores elevaciones de la muestra en Difusión de la Identidad (A), Autodevaluación (B) y Desaprobación Corporal (C); y elevaciones en Insensibilidad Social (F), Discordia Familiar (G) y Abuso Infantil (H). Un 84,6\% de ellas reporta violencia intrafamiliar en sus hogares y un $46,2 \%$ reconoce haber sido víctima de abuso sexual; además, refieren sintomatología variada, mala conducta y bajo rendimiento escolar, conductas agresivas $(76,9 \%)$ y rebeldía $(92,3 \%)$.

En Síndromes Clínicos destacan las elevaciones de Tendencia al Abuso de Sustancias (BB), Predisposición Delictual (CC) y Tendencia a la Impulsividad (DD). La elevación en $\mathrm{BB}$, junto a la configuración Transgresor-Poderoso-Predisposición a la Delincuencia, puede indicar que estas jóvenes usan o abusan de las drogas como un acto de desafío contra sus padres, para reducir inhibiciones y facilitar una actitud antisocial (McCann,1999). Concordantemente, en este grupo se encuentran las únicas mujeres que presentan conductas delictivas como motivo principal de consulta y el mayor consumo encontrado en la muestra de mujeres (ver Tabla 5).
Perfil tipo 2, mujeres Inhibidas Autodegradantes. Presentan muy elevada la configuración Introvertido-Inhibido-Afligido (12A-2B), que aparece en la Figura 5, lo cual señala que estas adolescentes se caracterizan por ser distantes, apáticas y retraídas socialmente, poseen una capacidad disminuida para experimentar placer y evidencian alta incomodidad cuando se enfrentan a dificultades. Además, las tres últimas elevaciones (8A, 8B y 9) indican que estas jóvenes presentan sufrimiento y gran inestabilidad emocional (McCann, 1999).

En Preocupaciones Expresadas se caracterizan por presentar dificultades en el desarrollo de una identidad madura (A), insatisfacción consigo mismas (B), desaprobación de sus características corporales, con la sensación subjetiva de escaso atractivo (C) y preocupaciones por relaciones familiares conflictivas (G). En Síndromes Clínicos, además de la esperable elevación en Tendencia al Abuso de Sustancias (BB), este grupo presenta elevaciones en Disfunciones de la Alimentación (AA), Tendencia a la Impulsividad (DD), Afecto Depresivo (FF) y Tendencia Suicida (GG). Esto sugiere que, además del consumo de drogas, estas adolescentes presentan bajo control de impulsos, sentimientos de tristeza, culpa y baja autoestima.

Congruentemente, este grupo informa en la entrevista sintomatología ansioso-depresiva (más del 90\% informa sentimientos de angustia, soledad y depresión), junto a una gran diversidad de otros síntomas, entre ellos, baja autoestima (90,9\%), dificultades

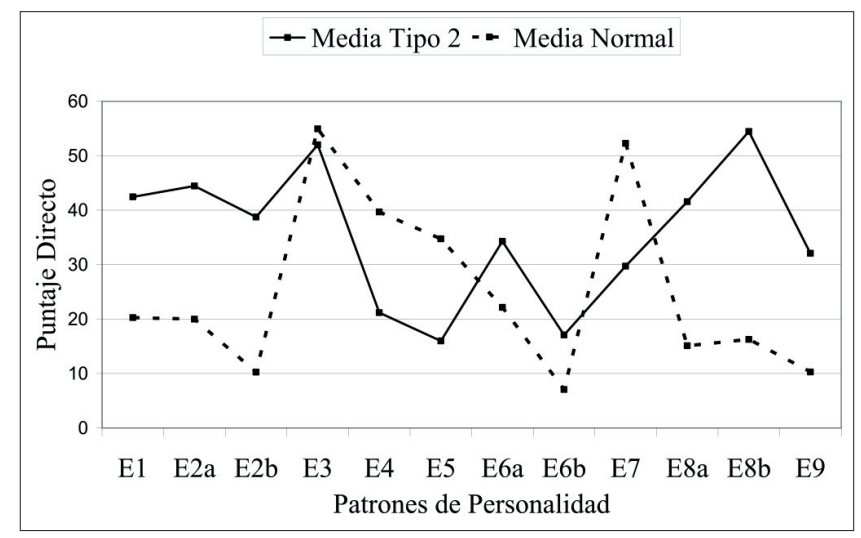

Figura 5. Perfil mujeres tipo 2. 
de concentración escolar (81,6\%), trastornos de la alimentación (72,7\%), desmotivación escolar $(63,6 \%)$ y violencia intrafamiliar en sus hogares (63,6\%). Entre los principales motivos de consulta aparecen ansiedad y/o depresión, quejas somáticas y consumo de drogas. Un 18,2\% presenta dependencia de sustancias y un $63,6 \%$, consumo habitual.

Perfil tipo 3, Subclínico mujeres. Al igual que el Tipo Subclínico hombres, este grupo presenta un perfil no patológico de personalidad, observándose la mayor elevación en Poderoso (6B), lo que indicaría que estas adolescentes pueden incurrir en conductas que violan los derechos y sentimientos de los demás (ver Figura 6).

En Preocupaciones Expresadas presentan elevaciones moderadas de Discordia Familiar (G) y Abuso Infantil (H) y, como es esperable, aparece una elevación signifi- cativa en Tendencia al Abuso de Sustancias (BB), aunque el consumo es de bajo nivel (ver Tabla 5). Es el grupo que presenta menor sintomatología, tanto en el MACI como en la entrevista.

Perfil tipo 4, mujeres en Riesgo. En comparación con los tipos 1 y 2 , las mujeres de tipo 4 presentan puntajes cercanos a lo normal (ver Figura 7), pero con riesgo de alteraciones debido a la elevación en algunas escalas.

En Preocupaciones Expresadas muestran elevaciones en las escalas Difusión de la Identidad (A) y Autodevaluación (B) y, al igual que otros tipos, presentan elevaciones en Tendencia al Abuso de Sustancias (BB), junto a elevaciones moderadas en Afecto Depresivo (FF) y Tendencia Suicida (GG), puntuaciones que potencian el riesgo de psicopatología.

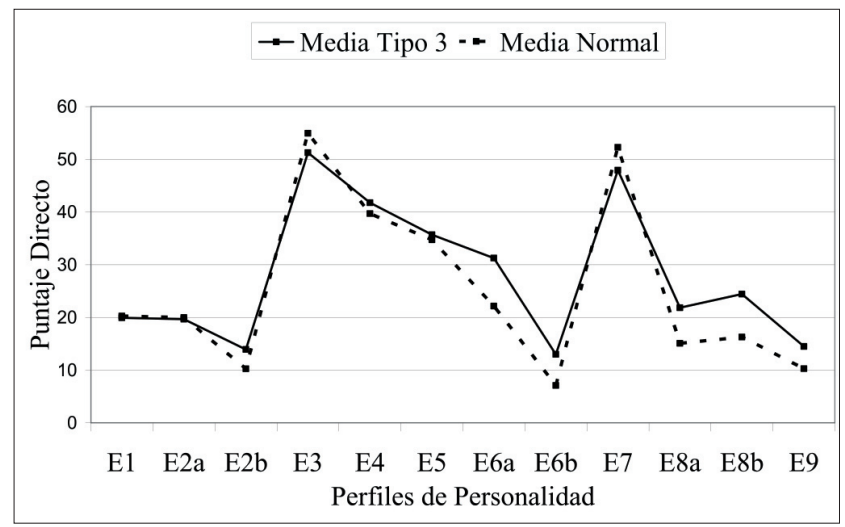

Figura 6. Perfil mujeres tipo 3.

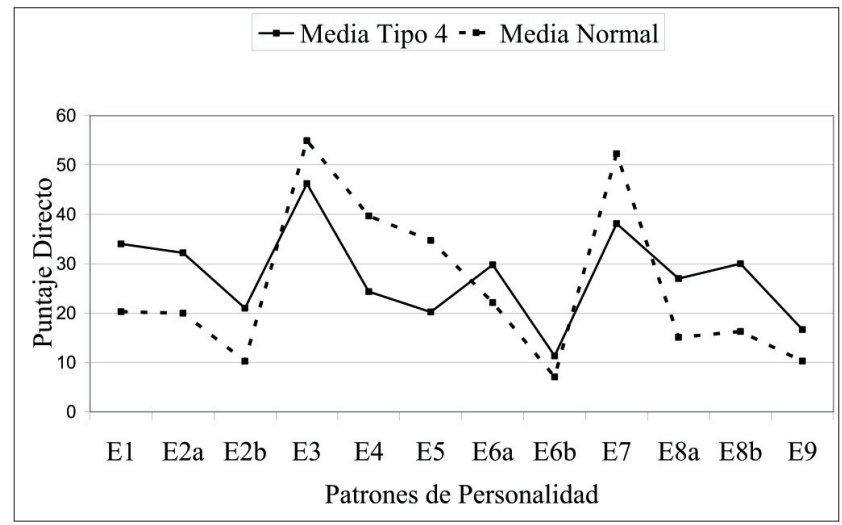

Figura 7. Perfil mujeres tipo 4. 
En la entrevista se aprecian alteraciones en el área escolar (cerca del 50\% presenta bajo rendimiento escolar, rechazo al estudio, dificultades de concentración y/o desmotivación), además presentan sintomatología emocional (sobre el $60 \%$ refiere angustia, baja autoestima, depresión y/o sentimientos de soledad). El motivo de consulta es variable, presentándose con frecuencia los problemas ansioso-depresivos (22,2\%). Presentan principalmente consumo no problemático $(77,8 \%)$, destacándose el consumo ocasional $(44,4 \%)^{2}$.

\section{Discusión}

Los principales resultados obtenidos en este estudio permiten afirmar, en primer lugar, que el MACI discrimina adecuadamente entre adolescentes consumidores de drogas y un grupo sin antecedentes de consumo de drogas. En la muestra de mujeres se encontraron diferencias significativas en 30 escalas del MACI y en la muestra de hombres se evidenciaron diferencias significativas en 28 de ellas. Estos resultados coinciden con los encontrados por Alarcón et al. (2004), Grilo et al. (1996), McCann (1997) y Romm et al. (1999), quienes señalan que el MACI permite una adecuada descripción del funcionamiento psicológico y conductual, diferenciando trastornos específicos, como los trastornos por uso de alcohol y drogas.

En general, se encontró que hombres y mujeres consumidores presentan puntajes más altos que el grupo no-consultante en la mayoría de las escalas MACI, excepto en las escalas Sumiso, Conformista y Sentimientos Ansiosos. Esto concuerda con los hallazgos de Grilo et al. (1996), quienes encontraron que los adolescentes estadounidenses hospitalizados con trastornos por consumo de sustancias presentaron puntajes bajos en las escalas Sumiso y Sentimientos Ansiosos y elevados en las escalas Predisposición Delictual, Transgresión e Insensibilidad Social, interpretando estos puntajes como reflejo de trastornos del área conductual.

\footnotetext{
2 La sistematización de la información obtenida en la entrevista se encuentra disponible para su revisión, previa solicitud a las autoras de este estudio.
}

Los puntajes bajos en las escalas Sumiso, Conformista y Sentimientos Ansiosos en los adolescentes chilenos consumidores de droga se asocian a características desafiantes de los valores y normas culturales, que pueden explicar conductas disruptivas, consumo de drogas ilícitas y abandono escolar, junto a menores niveles de ansiedad.

En Chile, Vinet y Alarcón (2003) encontraron en las escalas Sumiso, Conformista y Sentimientos Ansiosos puntajes más elevados en los adolescentes normales que en los clínicos o con desadaptación social, concluyendo que los adolescentes chilenos normales tendrían un estilo de afrontamiento a la realidad caracterizado por un mayor apego a los valores culturales tradicionales y también por vivencias de ansiedad acentuadas ante situaciones de conflicto, características que no estarían presentes en los adolescentes consumidores de drogas.

En segundo lugar, las escalas de Patrones de Personalidad del MACI permiten delimitar tipos de adolescentes consumidores de drogas, lo que coincide con la hipótesis de heterogeneidad en las características de personalidad de los consumidores de drogas, formulada por Eshbaugh, Tosi y Hoyt (1978).

Aunque las tipologías según género son mutuamente excluyentes entre sí, es posible señalar ciertas similitudes. Los hombres Transgresores-Poderosos y las mujeres Oposicionistas-Transgresoras tienen en común la configuración Transgresor-PoderosoOposicionista que, según McCann (1999), indicaría que estos adolescentes utilizan medios inconscientes para hacer frente a la tensión, presentando conductas hostiles, enojo y falta de relaciones interpersonales estrechas. También comparten los mayores niveles de consumo de drogas, los mayores porcentajes de conducta delictiva como motivo principal de consulta y elevaciones significativas en los puntajes de las escalas Tendencia al Abuso de Sustancia y Predisposición Delictual. Cabe destacar que las mujeres tipo 1 presentan un estilo transgresor de normas, más característico de los varones chilenos.

Diversas investigaciones sugieren que el abuso de sustancias ocurre con mayor frecuencia entre los adolescentes que 
tienen problemas psicológicos y del comportamiento, encontrándose co-morbilidad entre trastornos conductuales y consumo de drogas (Mirón, Serrano, Godás \& Rodríguez, 1997; Tarter, Laird, Kabene, Buckstein \& Kaminer, 1990). El hallazgo de los tipos hombres Transgresores-Poderosos y mujeres Oposicionistas-Transgresoras permiteafirmar que, entre los jóvenes evaluados, existe alta correlación entre consumo problemático de drogas y predisposición delictual, conductas transgresoras de normas y oposicionismo.

Los tipos hombres OposicionistasAutodegradantes, mujeres InhibidasAutodegradantes y mujeres OposicionistasAutodegradantes comparten la constelación Introvertido-Inhibido-Afligido que, según McCann (1999), identifican a adolescentes con una marcada dificultad para experimentar vivencias positivas o reforzadoras en su vida cotidiana. Son aislados socialmente, con expectativas negativas de las relaciones interpersonales estrechas y desmotivados en la búsqueda del placer. Comparten también la elevación en las escalas Inhibido e Inseguridad Grupal, las que permiten interpretar el consumo como una estrategia para hacer frente a altos niveles de ansiedad social o para ganar la aceptación de sus pares (McCann, 1999).

Por otro lado, el análisis de conglomerados, al igual que en el estudio de Alarcón et al. (2004), permitió señalar la existencia de tipos Subclínicos en hombres y mujeres. Estos adolescentes se caracterizan por estar adaptados al entorno y presentar tendencia a anular sus propios sentimientos y deseos para adoptar los valores y preceptos de los demás. Estos tipos indicarían que, al interior del grupo de adolescentes consumidores de drogas, existen jóvenes con personalidad normal, reforzando la idea de Eshbaugh et al. (1978) sobre la heterogeneidad en las características de personalidad de los consumidores de drogas.

Como en otras investigaciones (Alarcón et al., 2004; CONACE, 2002, 2004b, 2006; Kumpfer \& Turner, 1990; Novacek et al., 1991), se apreciaron diferencias en el consumo según género, observándose mayor consumo en los hombres. Según Alarcón et al. (2004), esta situación daría cuenta de una distribución natural de conductas de consumo en población adolescente. Por otra parte, se observa que la droga ilícita de mayor consumo en la muestra es la marihuana, coincidiendo con los datos de CONACE (2006).

Junto con confirmar la utilidad del MACI en el diagnóstico de adolescentes chilenos consumidores de drogas, este estudio apoya la hipótesis de heterogeneidad de las características de personalidad de los consumidores de drogas, aportando una tipología de adolescentes consumidores. Esta tipología permitirá diferenciar necesidades y orientar estrategias de intervención en las fases de prevención, diagnóstico y tratamiento. Las caracterizaciones de los diferentes tipos de consumidores obtenidas en esta investigación debieran invitar a los profesionales de la salud mental a realizar evaluaciones de personalidad de sus pacientes, pues la información que brindan puede ser de gran ayuda para la definición de objetivos y la determinación de estrategias terapéuticas.

Una limitación del estudio es la dificultad para generalizar los resultados a toda la población de adolescentes consumidores de drogas, pues la muestra utilizada no es representativa de toda la población. También es una limitación haber utilizado puntuaciones directas en el MACI, debido a que los baremos chilenos para el test aún no están publicados. No obstante, los resultados de este estudio son consistentes con la investigación empírica y los antecedentes teóricos recabados.

\section{Referencias}

Alarcón, A., Faúndez, X., Hita, C. \& Rodríguez, P. (2004). Caracterización de patrones de personalidad según MACI y de factores psicosociales de riesgo en un grupo de adolescentes consumidores de drogas de la ciudad de Temuco. Tesis no publicada para optar al grado de Licenciado en Psicología, Universidad de La Frontera, Temuco, Chile.

Alarcón, P., Vinet, E. \& Salvo, S. (2005). Estilos de personalidad y desadaptación social durante la adolescencia. Psykhe, 14(1), 3-16.

Álvarez, C. \& Restovic, M. F. (2003). Validez de constructo de los patrones de personalidad del MACI en adolescentes consultantes. Tesis no publicada para optar al grado de Licenciado en Psicología, Universidad de La Frontera, Temuco, Chile.

American Psychiatric Association (2002). Manual 
diagnóstico y estadístico de los trastornos mentales ( $4^{\mathrm{a}}$ ed.). Barcelona: Autor.

Ávila, A., Jiménez, F. \& González, M. (1996). Aproximación psicométrica a los patrones de personalidad y estilos de afrontamiento del estrés en la adolescencia: perspectivas conceptuales y técnicas de evaluación. En M. Casullo (Ed.), Evaluación psicológica en el campo de la salud (pp. 267-325). Buenos Aires: Paidós.

Becoña, E. (2002). Bases científicas de la prevención de las drogodependencias. Madrid: Ministerio del Interior. Extraído el 20 Septiembre, 2003, de http://www.pnsd.msc.es/Categoria2/publica/pdf/ Bases_cientificas.pdf

Consejo Nacional para el Control de Estupefacientes (2002). Quinto Estudio Nacional de Drogas en Población General de Chile. Santiago, Chile: Ministerio del Interior.

Consejo Nacional para el Control de Estupefacientes (2004a). Drogas: tratamiento y rehabilitación de niños, niñas y adolescentes. Santiago, Chile: Ministerio del Interior.

Consejo Nacional para el Control de Estupefacientes (2004b). Sexto Estudio Nacional de Drogas en Población General de Chile. Santiago, Chile: Ministerio del Interior.

Consejo Nacional para el Control de Estupefacientes (2006). Séptimo Estudio Nacional de Drogas en Población General de Chile. Santiago, Chile: Ministerio del Interior.

Consejo Nacional para el Control de Estupefacientes (2008). Séptimo Estudio Nacional de Drogas en Población Escolar de Chile. Santiago, Chile: Ministerio del Interior.

Díaz, C. (2003). Desarrollo de una tipología de adolescentes consultantes en salud mental, a partir del Inventario Clínico para Adolescentes de Millon. Tesis no publicada para optar al grado de Magíster en Psicología, Universidad de La Frontera, Temuco, Chile.

Eshbaugh D. M., Tosi, D. J. \& Hoyt, Ch. (1978). Some personality patterns and dimensions of male alcoholics: A multivariate description. Journal of Personality Assessment, 42, 409-417.

Grilo, C., Fehon, D., Walker, M. \& Martino, S. (1996). A comparison of adolescents' inpatients with and without substance abuse using the Millon Adolescent Clinical Inventory. Journal of Youth and Adolescence, 25, 379-389.

Hair, J. F. \& Anderson, R. E. (1999). Análisis multivariante. Madrid: Prentice Hall Iberia.

Kumpfer, K. L. \& Turner, Ch. W. (1990). The Social Ecology Model of adolescent substance abuse: Implications for prevention. International Journal of the Addictions, 25(4), 435-463.

McCann, J. (1997). The MACI: Composition and clinical application. En T. Millon (Ed.), The Millon inventories (pp. 363-388). New York: Guilford.

McCann, J. (1999). Using the Millon Adolescent Clinical Inventory. Assessing adolescent with the $M A C I$. New York: Wiley and Sons.

Millon, T. (1993). Manual of Millon Adolescent Clinical Inventory. Minneapolis, MN: National Computer Systems.

Millon, T. (1998). El modelo de polaridad de Millon. En T. Kirchner, M. Torres \& M. Forns (Eds.), Evaluación psicológica: modelos y técnicas (pp. 241-266). Barcelona: Paidós.

Mirón, L., Serrano, G., Godás, A. \& Rodríguez, D. (1997). Conducta antisocial y consumo de drogas en adolescentes españoles. Análisis y Modificación de Conducta, 23, 255-282.

Novacek, J., Raskin, R. \& Hogan, R. (1991). Why do adolescents use drugs? Age, sex, and user differences. Journal of Youth and Adolescence, 20, 475-492.

Romm, S., Bockian, N. \& Harvey, M. (1999). Factorbased prototypes of the Millon Adolescent Clinical Inventory in adolescents referred for residential treatment. Journal of Personality Assessment, 72, 125-143.

Stefurak, T., Calhoun, G. B. \& Glaser, B. A. (2004). Personality typologies of male juvenile offenders using a cluster analysis of the Millon Adolescent Clinical Inventory Introduction. International Journal of Offender Therapy and Comparative Criminology, 48, 96-110.

Tarter, R., Laird, S., Kabene, M., Buckstein, O. \& Kaminer, Y. (1990). Drug abuse severity in adolescents is associated with magnitude of deviation in temperament traits. British Journal of Addiction, 85, 1501-1504.

Taylor, J., Kemper, T. S., Loney, B. R. \& Kistner, J. A. (2006). Classification of severe male juvenile offenders using the MACI Clinical and Personality Scales. Journal of Clinical Child and Adolescent Psychology, 35, 90-102.

United Nations, Office on Drugs and Crime (2008). Annual Report 2008. Extraído el 30 Septiembre, 2008, de http://www.unodc.org/documents/aboutunodc/AR08_WEB.pdf

Vinet, E. \& Alarcón, P. (2003). El MACI en la evaluación de adolescentes chilenos. Psykhe, 12(1), 39-55.

Vinet, E., Brió, C., Correa, P., Díaz, P., Diez, M., Echeverría, M., Salazar, D. \& Vargas, A. (1999). MACI Traducción y adaptación chilena para uso exclusivo en investigación (DIDUFRO 9966). Manuscrito no publicado, Universidad de La Frontera, Temuco, Chile.

Vinet, E., Salvo, S. \& Forns, M. (2005). Adolescentes no consultantes en riesgo: una evaluación a través del MACI. Anuario de Psicología, 36, 83-97.

Fecha de recepción: Julio de 2008.

Fecha de aceptación: Marzo de 2009. 